\title{
Estudio de la granulación de la mezcla de minerales de hierro en el proceso de sinterización. Il parte. Índice de granulación ${ }^{(\cdot)}$
}

\author{
Antonio Formoso*, Alfredo Moro**, Gerardo Fernández-Pello***, Moisés Muñiz***, \\ Juan Jiménez*, Aníbal Moro* y Alejandro Cores*
}

\begin{abstract}
Resumen Se estudia la aptitud a la granulación de una mezcla mineral utilizada para la fabricación de una serie de sinterizados en planta piloto. Se determinan los índices de calidad y la composición de las fases de los sinterizados. Se comprueba que se han obtenido sinterizados con estructura óptima. Se clasifica una serie de minerales de hierro según el índice de granulación (índice $\mathrm{G})$. Se ofrece la evolución durante los últimos años del índice $\mathrm{G}$ de las mezclas minerales utilizadas en ACERALIA y se comprueba cómo aumenta la productividad y disminuye el consumo de coque en la banda de sinterización con la mejora del índice G.
\end{abstract}

Palabras clave Granulación. Índice de granulación. Sinterización. Horno alto. Minerales de hierro.

\section{Study of the iron ores mixture granulation in the sintering process. Part 2. Granulation index}

\begin{abstract}
The fitness for granulation of a ore mixture used to make a series of sintering in a pilot plant is studied. The quality indexes and the phases composition of sinterings is determined. The best sintering structure is checked. A serie of iron ores is classified as the granulation index ( $G$ index). The $G$ index evolution of the ore mixtures used by ACERALIA for the last years is shown. An increase in productivity and a decrease in coke consumption in the sinter strand is observed as the $G$ index improves.
\end{abstract}

Keywords Granulation. Granulation index. Sintering. Blast furnace. Iron ores.

\section{FABRICACIÓN DE SINTERIZADOS EN PLANTA PILOTO}

La instalación usada para sinterizar una serie de diferentes mezclas minerales, utilizando distintas proporciones de los minerales de hierro indicados en la tabla I (I parte), según el trabajo ref. 26 de la I parte, es una planta piloto que el CENIM tiene instalada en ACERALIA en Gijón (Fig. 1), con una paila de $40 \times 40 \mathrm{~cm}$ de sección transversal y 60 $\mathrm{cm}$ de altura, equipada con un circuito de aspiración capaz de crear depresiones superiores a 1.500 $\mathrm{mm} \mathrm{H}_{2} \mathrm{O}$, un sistema de encendido de propano y un sistema de medida del volumen de aire de entrada y de la temperatura de gas de salida. Esta planta se

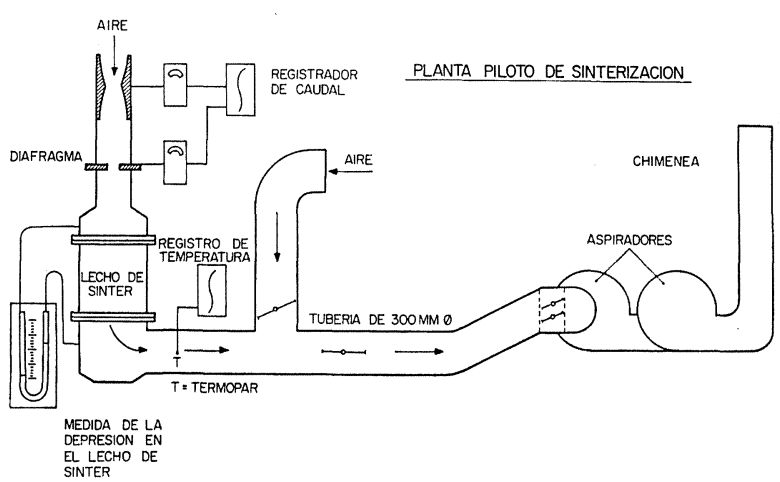

Figura 1. Planta piloto de sinterización.

Figure 1. Sintering pilot plant.

(•) Trabajo recibido el día 27 de marzo de 2000 y aceptado en su forma final el 19 de julio de 2000.

(*) Centro Nacional de Investigaciones Metalúrgicas, CENIM (CSIC). Avda. de Gregorio del Amo, 8. 28040 Madrid (España).

(**) Escuela de Ingeniería Técnica de Minas. Mieres. Universidad de Oviedo (España).

${ }^{(* * *)}$ ACERALIA Corporación Siderúrgica. Gijón, Asturias (España). 
utiliza por ACERALIA-CENIM para realizar trabajos de investigación conjunta ${ }^{[1-4]}$.

\subsection{Composición de la mezcla mineral 2}

En la tabla I se ofrece el porcentaje de los componentes de la mezcla mineral 2. Esta mezcla se ha elegido entre las utilizadas (ref. 26 de la I parte) porque ha sido la que ha dado origen a la fabricación de sinterizados de mejor calidad.

Tabla I. Mezcla mineral 2, \% en masa

Table l. Ore mixture 2, \% mass

\begin{tabular}{lrlr}
\hline Andaluza & 10,3 & MBR Brasil & 10,3 \\
CVRD-Carajas & 27,8 & Escoria LD & 3,0 \\
San Isidro & 7,2 & Chatarra & 1,3 \\
Hamersley & 12,4 & Polvo de tragante & 0,7 \\
SNIM-Tazadit & 10,3 & Lodos LD & 2,6 \\
SNIM-Normal & 6,2 & Finos de retorno & 7,9 \\
\hline
\end{tabular}

\subsubsection{Análisis químico de la mezcla}

En la tabla II se indica el análisis químico. La ley en hierro es alta como corresponde a los minerales presentes. Los contenidos en álcalis y ferroso son bajos.

\subsubsection{Análisis granulométrico de la mezcla}

En la figura 2 se representa la curva granulométrica de la mezcla. Este análisis se ha realizado por duplicado sobre muestra seca. En la figura se pueden ver los porcentajes de las diferentes fracciones granulométricas expresadas por su valor medio; se reflejan también las fracciones acumuladas. El tamaño medio de la mezcla es de $1,90 \mathrm{~mm}$.

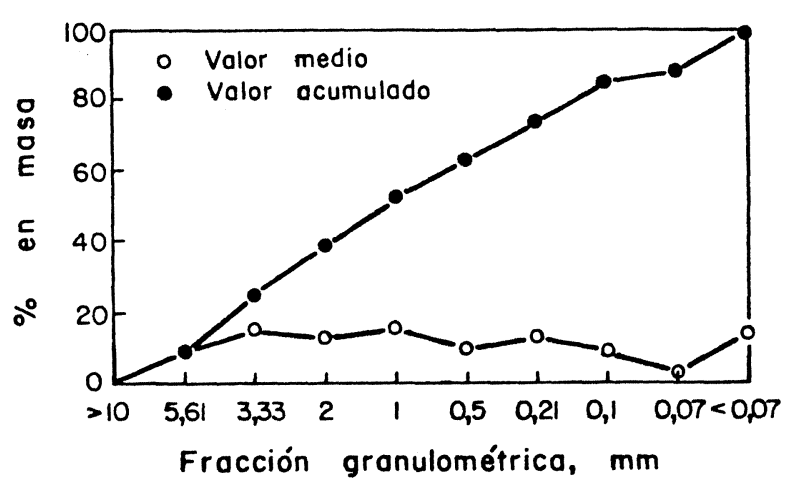

Figure 2. Curva granulométrica de la mezcla de mineral 2.

Figure 2. Granulometric curve of ore mixture 2.
Tabla II. Análisis químico de la mezcla mineral 2, \% en masa Table II. Ore mixture 2, \% mass

\begin{tabular}{lrll}
\hline Fe total & 60,28 & $\mathrm{Na} 2 \mathrm{O}$ & 0,015 \\
$\mathrm{Fe}^{++}$ & 3,59 & $\mathrm{~K} 2 \mathrm{O}$ & 0,054 \\
$\mathrm{CaO}$ & 4,57 & $\mathrm{~Pb}$ & 0,015 \\
$\mathrm{MgO}$ & 0,44 & $\mathrm{Zn}$ & 0,044 \\
$\mathrm{Al}_{2} \mathrm{O}_{3}$ & 1,04 & $\mathrm{~S}$ & 0,023 \\
$\mathrm{SiO}_{2}$ & 3,46 & $\mathrm{P} 2 \mathrm{O} 5$ & 0,082 \\
$\mathrm{MnO}$ & 0,69 & P.C. & 4,41 \\
\hline
\end{tabular}

P.C. = Pérdidas por calcinación.

\subsubsection{Aptitud a la granulación de la mezcla}

En la figura 3 se representa la curva granulométrica de la mezcla inicial y después del ensayo SAFE.

La mezcla presenta buena aptitud a la granulación, como era de esperar debido a los minerales que forman parte de su composición. El tamaño medio de la mezcla en crudo es de 1,90 mm pasando a 3,39 $\mathrm{mm}$ al final de la granulación.

\subsection{Fabricación de los sinterizados}

En la planta piloto (Fig. 1) se ha fabricado una serie de sinterizados con la mezcla mineral 2 (Tabla I). Para la fabricación se han mantenido unos parámetros de operación fijos, de acuerdo con la práctica industrial, como son:

- depresión de trabajo: $1.500 \mathrm{~mm} \mathrm{H}_{2} \mathrm{O}$

- aporte calorífico en el encendido: $12.480 \mathrm{~kJ}$

- tiempo de encendido: 1,5 min

- altura de sobreparrilla: $3 \mathrm{~cm}$

y unos parámetros variables:

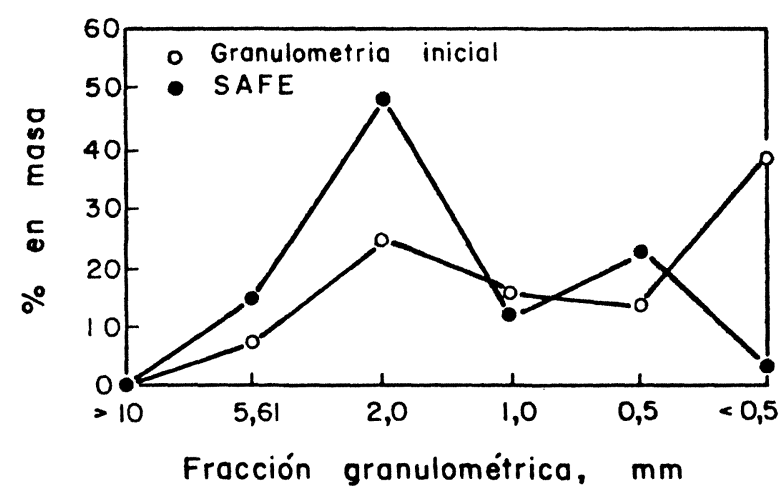

Figura 3. Aptitud a la granulación de la mezcla mineral 2.

Figure 3. Granulation fitness of ore mixture 2. 
- altura de lecho: 50 y $60 \mathrm{~cm}$

- finos de retorno: 25 y $35 \%$

- índice de basicidad $\left(\mathrm{CaO} / \mathrm{SiO}_{2}\right): 1,6$ y 1,9

En la tabla III se ofrecen las variables de operación y las características de los sinterizados fabricados.

El peso total de la mezcla que se carga en la paila aumenta con la altura del lecho de 170 a $210 \mathrm{~kg}$.

En la tabla se puede ver el peso de las distintas fracciones granulométricas de los sinterizados, con un tamaño medio que varía de 30 a $42 \mathrm{~mm}$. El consumo de coque oscila entre 39 y $44 \mathrm{~kg} / \mathrm{t}$ de sínter. La productividad se mantiene entre 37 y $42 \mathrm{t} / \mathrm{m}^{2} \cdot 24 \mathrm{~h}$.

De la comparación de los resultados obtenidos se podrán fijar aquellas variables para fabricar los sinterizados de mejor calidad, para la mezcla mineral estudiada.

\subsubsection{Análisis químico de los sinterizados}

En la tabla IV se ofrece el análisis químico de los sinterizados. La ley en hierro es alta y los contenidos de los elementos nocivos, azufre, fósforo y álcalis son bajos; por ello son una carga adecuada para su consumo en el horno alto.

\subsubsection{Ensayo RDI}

La degradación del sinterizado durante la reducción a baja temperatura se determina por el ensayo $R I^{[5]}$. El RDI de los sinterizados fabricados oscila entre un valor que se considera bueno de 40,9 hasta un valor máximo de 48,9 (Tabla V). Es deseable valores inferiores a 40 para este índice. La degradación del sinterizado tiene lugar durante la reducción en el horno alto, en la zona de baja

Tabla III. Sinterizados fabricados en planta piloto con la mezcla mineral 2

Table III. Sinterings made in pilot plant with the ore mixture 2

\begin{tabular}{|c|c|c|c|c|c|c|c|c|}
\hline Sinterizado, núm. & S18 & $\mathrm{S} 20$ & $\mathrm{~S} 22$ & S24 & S26 & S28 & S30 & S32 \\
\hline Altura de lecho, $\mathrm{cm}$ & 50 & 50 & 50 & 50 & 60 & 60 & 60 & 60 \\
\hline Finos de retorno, $\%$ & 25 & 25 & 35 & 35 & 25 & 25 & 35 & 35 \\
\hline Basicidad, $\mathrm{CaO} / \mathrm{SiO}_{2}$ & 1,6 & 1,9 & 1,6 & 1,9 & 1,6 & 1,9 & 1,6 & 1,9 \\
\hline Mezcla mineral $2, \mathrm{~kg}$ & 112,3 & 112,3 & 112,3 & 112,3 & 138,1 & 138,1 & 138,1 & 138,1 \\
\hline Dunita, kg & 2,2 & 2,0 & 2,0 & 1,9 & 2,8 & 2,7 & 2,3 & 2,3 \\
\hline Coque, kg & 5,0 & 4,8 & 4,4 & 4,5 & 5,8 & 5,9 & 5,5 & 5,5 \\
\hline Finos de retorno, kg & 27,5 & 27,5 & 38,5 & 38,5 & 33,8 & 33,8 & 47,3 & 47,3 \\
\hline Caliza, kg & 10,1 & 13,1 & 8,9 & 12,9 & 11,9 & 16 & 10,8 & 15,6 \\
\hline Agua, kg & 8,5 & 8,7 & 9,0 & 9,5 & 11,0 & 11,0 & 11,5 & 11,9 \\
\hline Total peso mezcla, kg & 165,6 & 168,4 & 175,1 & 179,6 & 203,4 & 207,5 & 215,5 & 220,7 \\
\hline \multicolumn{9}{|l|}{ Granulometría, mm } \\
\hline$>40, \mathrm{~kg}$ & 43,7 & 38,0 & 38,9 & 36,7 & 57,6 & 42,0 & 55,1 & 50,0 \\
\hline $40-20, \mathrm{~kg}$ & 27,3 & 33,2 & 20,8 & 24,8 & 27,4 & 37,8 & 23,3 & 27,6 \\
\hline $20-12, \mathrm{~kg}$ & 9,9 & 11,2 & 7,8 & 9,2 & 11,5 & 14,5 & 8,5 & 10,1 \\
\hline $12-5, \mathrm{~kg}$ & 25,8 & 24,3 & 31,9 & 29,2 & 34,6 & 33,7 & 34,4 & 34,2 \\
\hline$<5, \mathrm{~kg}$ & 25,2 & 24,8 & 37,2 & 33,7 & 33,8 & 32,6 & 46,5 & 41,5 \\
\hline Total peso torta, $\mathrm{kg}$ & 131,9 & 131,4 & 136,6 & 133,5 & 164,8 & 160,6 & 167,7 & 163,4 \\
\hline Sínter útil, kg & 106,7 & 106,6 & 99,4 & 99,8 & 131,0 & 128,0 & 121,3 & 121,9 \\
\hline Finos de retorno, $\mathrm{kg}$ & 25,2 & 24,8 & 37,2 & 33,7 & 33,8 & 32,6 & 46,5 & 41,5 \\
\hline Tamaño medio, mm & 33,8 & 32,8 & 29,6 & 29,7 & 41,8 & 37,5 & 38,8 & 37,8 \\
\hline Densidad de carga, $\mathrm{kg} / \mathrm{m}^{3}$ & 1.949 & 1.957 & 1.983 & 1.957 & 2.011 & 1.971 & 1.996 & 1.964 \\
\hline Depresión inicial, $\mathrm{mm} \mathrm{H}_{2} \mathrm{O}$ & 820 & 880 & 800 & 800 & 880 & 880 & 940 & 920 \\
\hline Depresión total, $\mathrm{mm} \mathrm{H}_{2} \mathrm{O}$ & 1.500 & 1.500 & 1.500 & 1.500 & 1.500 & 1.500 & 1.500 & 1.500 \\
\hline Temp. máxima humos, ${ }^{\circ} \mathrm{C}$ & 350 & 370 & 380 & 380 & 340 & 380 & 400 & 380 \\
\hline Permeabilidad, $\mathrm{min} / \mathrm{s}$ & 2,01 & 2,04 & 2,02 & 2,02 & 2,02 & 1,57 & 1,55 & 1,53 \\
\hline Consumo coque, $\mathrm{kg} / \mathrm{t}$ de sínter & 44,2 & 42,0 & 40,2 & 39,4 & 42,8 & 42,8 & 41,0 & 39,2 \\
\hline Productividad, $\mathrm{t} / \mathrm{m}^{2} \cdot 24 \mathrm{~h}$ & 39,6 & 39,4 & 37,2 & 37,2 & 40,5 & 41,8 & 34,9 & 36,4 \\
\hline
\end{tabular}


Tabla IV. Análisis químico de los sinterizados, \% en masa

Table IV. Chemical analysis of sinterings, \% mass

\begin{tabular}{lcccccccc}
\hline & S18 & S20 & S22 & S24 & S26 & S28 & S30 & S32 \\
\hline Fe total & 57,3 & 56,6 & 57,6 & 56,9 & 58,0 & 56,5 & 57,6 & 57,1 \\
$\mathrm{FeO}$ & 5,7 & 5,6 & 3,7 & 3,6 & 4,7 & 5,0 & 3,5 & 3,9 \\
$\mathrm{SiO}_{2}$ & 5,4 & 5,3 & 5,4 & 4,9 & 5,2 & 5,7 & 5,0 & 5,8 \\
$\mathrm{Al}_{2} \mathrm{O}_{3}$ & 1,22 & 1,17 & 1,19 & 1,16 & 1,20 & 1,10 & 1,19 & 1,10 \\
$\mathrm{CaO}$ & 9,35 & 10,50 & 8,80 & 10,35 & 8,60 & 10,13 & 9,05 & 9,20 \\
$\mathrm{MgO}$ & 1,70 & 1,68 & 1,60 & 1,61 & 1,62 & 1,66 & 1,56 & 1,45 \\
$\mathrm{MnO}$ & 0,74 & 0,72 & 0,80 & 0,71 & 0,75 & 0,73 & 0,79 & 0,69 \\
$\mathrm{~S}$ & 0,011 & 0,014 & 0,013 & 0,018 & 0,012 & 0,014 & 0,012 & 0,015 \\
$\mathrm{P}$ & 0,042 & 0,039 & 0,040 & 0,040 & 0,040 & 0,036 & 0,041 & 0,038 \\
$\mathrm{Na}$ & 0,039 & 0,037 & 0,036 & 0,031 & 0,032 & 0,036 & 0,040 & 0,039 \\
$\mathrm{~K}_{2} \mathrm{O}$ & 0,071 & 0,071 & 0,072 & 0,068 & 0,066 & 0,072 & 0,075 & 0,072 \\
$\mathrm{CaO} / \mathrm{SiO}_{2}$ & 1,73 & 1,98 & 1,64 & 2,13 & 1,67 & 1,82 & 1,81 & 1,59 \\
\hline
\end{tabular}

temperatura, $850-950^{\circ} \mathrm{C}$, y causa un efecto perjudicial sobre la resistencia de la carga en el horno, con la subsiguiente pérdida de permeabilidad de los gases reductores y aumento del consumo de coque $^{[6]}$.

La degradación se origina en la transformación, hasta una cierta extensión, que tiene lugar durante la reducción de hematita a magnetita, que va acompañada de un aumento de volumen dando lugar a la presencia de tensiones estructurales en el sinterizado ${ }^{[7]}$.

La degradación se ha relacionado con el contenido de hematita secundaria en el sinterizado (ref. 15 de la I parte). Se encontró que las grietas en la estructura de los sinterizados investigados eran más abundantes donde había presencia local de tales granos de hematita, siendo estas grietas perjudiciales para el RDI.

\subsubsection{Ensayo Tumbler}

La resistencia en frío del sinterizado se determina por el ensayo Tumbler ${ }^{[8]}$ y depende de las resistencias de los componentes minerales individuales, la resistencia del constituyente matriz enlazante y la composición del mineral. El estudio de la resistencia a la fractura de varias fases minerales ha dado lugar al orden siguiente ${ }^{[9]}$ : hematita primaria > hematita secundaria $>$ magnetita $>$ SFCA $>$ vidrio.

En un estudio se ha podido relacionar la resistencia de los gránulos sinterizados con la fluidez del fundido formado por la capa de partículas adherentes al núcleo. Los factores principales que afectan a la resistencia son la composición química de la capa adherente, el tamaño del poro y la pérdida por calcinación de las partículas del núcleo ${ }^{[10]}$. También se ha ęstudiado que la resistencia está relacionada con la fracción de huecos y con el área superficial específica de la torta del sinterizado ${ }^{[11]}$.

En la tabla $\mathrm{V}$ se puede observar que los sinterizados presentan buenos resultados para el índice Tumbler, que en algunos casos es superior a 70 .

\subsubsection{Ensayo de reductibilidad ISO}

La reductibilidad del sinterizado se determina mediante el ensayo ISO que se realiza a $900^{\circ} \mathrm{C}$ (ref. 8 de la I parte). Se han llevado a cabo investigaciones con el fin de aumentar la reductibilidad del sinterizado, lo que ocasiona una mejora en la estabilidad y un ahorro en el consumo de coque en el horno alto. La reductibilidad está muy relacionada con la estructura y porosidad del sinterizado. La reductibilidad de las fases minerales disminuyen en el orden:

$$
\mathrm{Fe}_{2} \mathrm{O}_{3}>\mathrm{CaO} \cdot 2 \mathrm{Fe}_{2} \mathrm{O}_{3}>\mathrm{CaO} \cdot \mathrm{Fe}_{2} \mathrm{O}_{3}>2 \mathrm{CaO} \cdot \mathrm{Fe}_{2} \mathrm{O}_{3}>\mathrm{Fe}_{3} \mathrm{O}_{4}
$$

La hematita y magnetita se reducen rápidamente a wustita ( $\mathrm{FeO})$, pero las velocidades difieren para la posterior reducción a hierro metálico ${ }^{[12]}$. A partir de la hematita, la wustita se reduce de forma homogénea y rápida, aunque alguna wustita se encuentra rodeada de metal. A partir de la magnetita, la reducción es topoquímica, según la secuencia $\mathrm{Fe}_{3} \mathrm{O}_{4} \rightarrow \mathrm{FeO} \rightarrow \mathrm{Fe}$, y casi todos los granos de magnetita son rodeados rápidamente de hierro metálico, que retarda la reducción posterior.

La reductibilidad de SFCA se puede relacionar con su morfología, su porosidad y si está recubierto 
de vidrio (ref. 17 de la I parte). El ferrito acicular, formado a baja temperatura de sinterización, es más reductible, mientras que el ferrito columnar, formado a alta temperatura (posiblemente recubierto de vidrio) es menos reductible. La hematita primaria es más reductible que la hematita secundaria, a causa de su porosidad intrínseca.

Se ha investigado el efecto de la porosidad sobre la reductibilidad y se observa que el ferrito estabiliza la presencia de poros finos que se producen durante la reducción del sinterizado, conduciendo a aumentar la porosidad y a una reductibilidad más elevada. El uso del mineral goethítico, muy poroso, también favorece la reductibilidad.

En la tabla V se puede ver que los sinterizados presentan un buen comportamiento en la reducción, con valores que se encuentran en el intervalo $78-86 \%$.

\subsubsection{Caracterización de la estructura por micros- copía electrónica}

Dada la diversidad de los componentes mineralógicos que forman parte de la mezcla mineral, así como de su propia heterogeneidad, se comprende que la estructura del sinterizado sea también compleja, estando formada principalmente por granos de óxido de hierro y de ferrito cálcico ligados por una matriz de ganga ${ }^{[13 \text { y } 14]}$. Los ferritos, cuya cantidad aumenta con el índice de basicidad, son de fácil reducción, y por aumentar la resistencia mecánica del sinterizado, hasta ciertos niveles, se consideran constituyentes muy beneficiosos para la estructura. La ganga está constituida por silicatos de calcio, hierro y magnesio, difíciles de reducir, y en el horno alto pasan a formar parte de la escoria.

Las muestras de los sinterizados se han estudiado en un microscopio electrónico de barrido con microsonda JEOL, con un analizador LINK AN10000.

Para la observación microscópica, la probeta de cada muestra de sinterizado se prepara según las etapas siguientes:

- Partiendo de la muestra representativa (12,5$10,0 \mathrm{~mm}$ ) por cuarteo se toma una fracción de $200 \mathrm{~g}$

- Se muele la muestra en un mortero manual y se criba por los tamices de 1,0 y $0,35 \mathrm{~mm}$. La fracción obtenida entre ambos tamices (1,0-0,35 $\mathrm{mm}$ ) será la granulometría utilizada para la observación microscópica

- Las partículas de esta fracción se embeben en una resina, que luego se corta, obteniéndose probetas cilíndricas de $30 \mathrm{~mm}$ de diámetro y unos $10 \mathrm{~mm}$ de altura.

Se ha realizado un número considerable de fotos de aspectos generales y en detalle de los sinterizados S 22, S 28 y S 30, elegidos como representativos.

En la figura 4 se ofrece el aspecto de un grupo de partículas de sinterizado S 30. Al hacer la exploración de la muestra se observa que un número elevado de partículas contienen ferritos en su composición. En la figura 5 se ofrece con detalle una zona de la partícula P 53, con presencia de una red de ferritos aciculares y columnares, ganga y porosidad.

La figura 6 ofrece detalle de la P 54, con una estructura óptima de sinterizado formada por un

Tabla V. Ensayos RDI, Tumbler y de reductibilidad

Table V. RDI, Tumbler and reductibility test

\begin{tabular}{cccccc}
\hline \multirow{2}{*}{ Sínter } & RDI & Tumbler & & \multicolumn{3}{c}{ Reductibilidad } \\
\cline { 4 - 6 } & $(<3 \mathrm{~mm})$ & $(>6,3 \mathrm{~mm})$ & $\begin{array}{c}\text { Velocidad de } \\
\text { reducción } \\
(\mathbf{d R / d t})_{40} \\
\left(\% \mathrm{O}_{2} / \mathrm{min}\right)\end{array}$ & $\begin{array}{c}\text { Grado de } \\
\text { reducción } \\
\mathbf{R}_{60}(\%)\end{array}$ & $\begin{array}{c}\text { Grado de } \\
\text { metalización } \\
(\%)\end{array}$ \\
\hline S18 & 48,9 & 66,7 & 1,56 & 79,10 & 79,70 \\
S20 & 45,3 & 70,0 & 1,60 & 86,31 & 80,26 \\
S22 & 41,0 & 60,0 & 1,60 & 80,89 & 74,90 \\
S24 & 45,7 & 64,0 & 1,60 & 82,29 & 80,93 \\
S26 & 40,9 & 67,3 & 1,40 & 78,23 & 68,43 \\
S28 & 42,0 & 72,7 & 1,49 & 80,52 & 75,66 \\
S30 & 47,6 & 58,0 & 1,56 & 81,53 & 80,32 \\
S32 & 45,3 & 66,7 & 1,68 & 83,99 & 80,41 \\
\hline
\end{tabular}




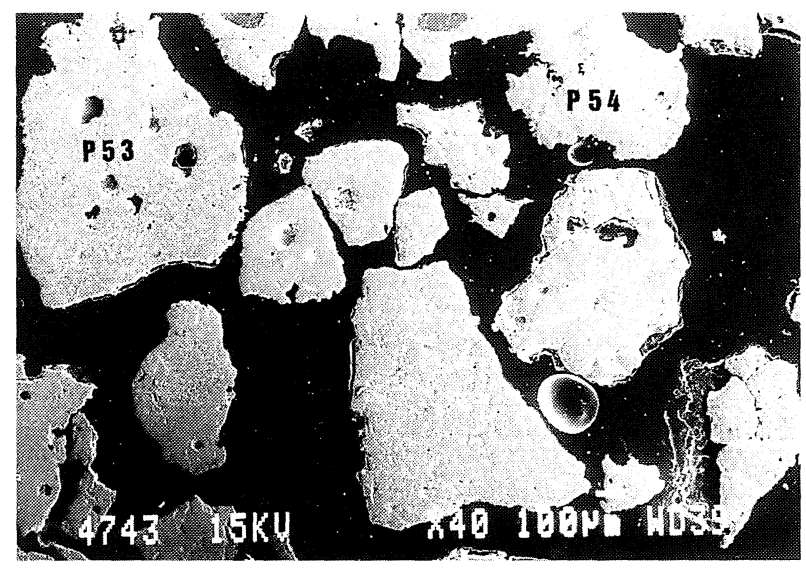

Figura 4. Aspecto general de partículas del sinterizado $S$ $30(x 40)$.

Figure 4. General appearance of sintering $S 30$ particles (40x).

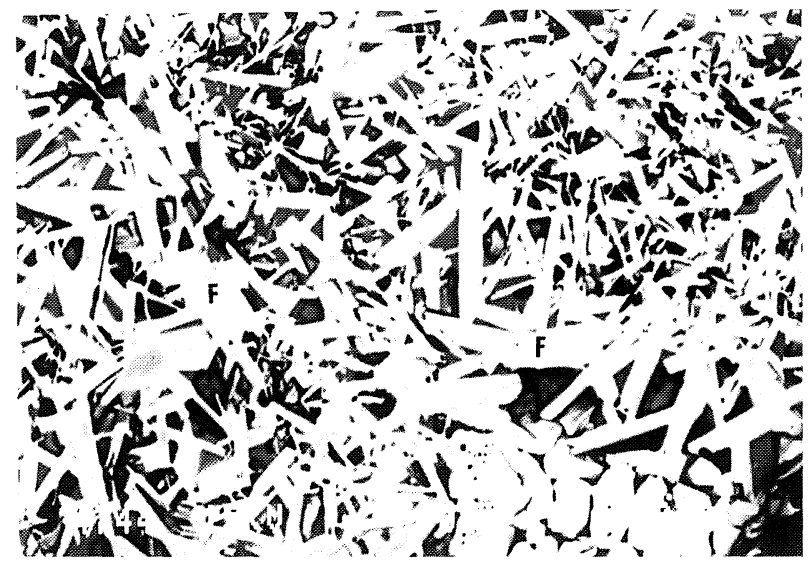

Figura 5. Detalle de la partícula $P$ 53, localizda en la figura 4 , con presencia de una red de ferritos $(x 800) . F=$ Red de ferritos. Presencia de ganga (gris oscuro) y poros (negrol.

Figura 5. Close view of particle $P$ 53, localised in figure 4, with presence of a lattice of ferrites $(800 x) . F=$ Lattice of ferrites. Presence of gangue (dark grey) and pores (black).

núcleo de hematita rodeado de una red de ferritos aciculares. La figura 7 corresponde a una zona de la partícula P 57, no localizada en la figura 4, con estructura óptima de sinterizado.

El análisis de las fases identificadas se ofrece en la tabla VI. El contenido en oxígeno se calcula por diferencia hasta 100 de la suma de los demás elementos.

\section{2. ÍNDICE G}

La clasificación de los minerales de hierro que forman parte de una mezcla mineral utilizada en el proceso de sinterización permitirá predecir el tipo de sinterizado obtenido.

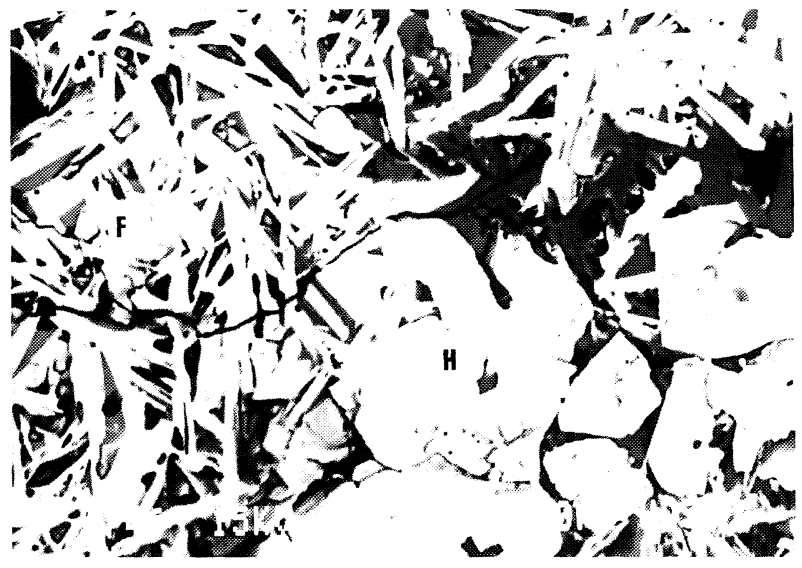

Figura 6. Detalle de la partícula P 54 , localizada en la figura 4 , con estructura óptima de sinterizado $(\times 900) . \mathrm{H}=$ Hematita; $F=$ Red de ferritos. Presencia de ganga (gris oscuro) y poros (negro).

Figure 6. Close view of particle P 54, localised in figure 4, with the best sinterized structure (900x). H. Hematite; $F=$ Lattice of ferrites. Presence of gangue (dark grey) and pores (black).

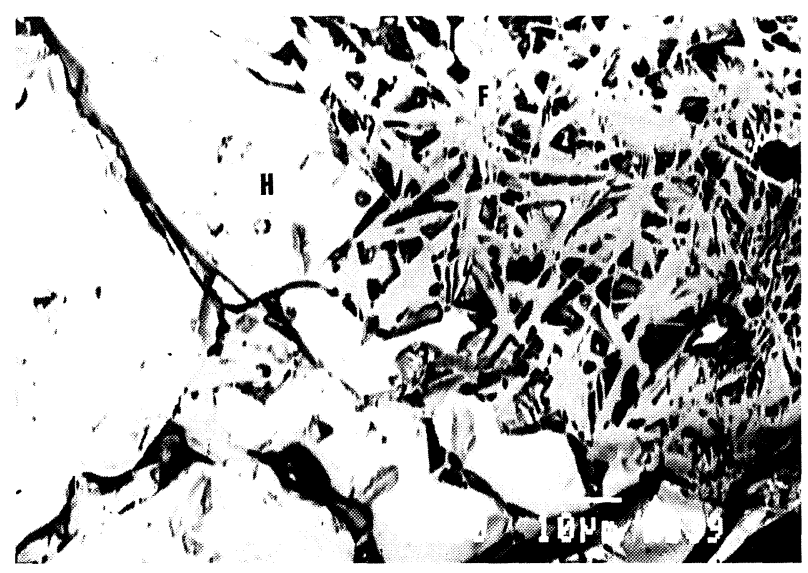

Figura 7. Detalle de la partícula $P 57$ con estructura óptima de sinterizado $(x 750) . \mathrm{H}=$ Hematita; $F=$ Red de ferritos. Presencia de ganga (gris oscuro) y porosidad (negro).

Figure 7. Close view of particle $P$ 54, with the best sinterized structure $(750 x)$. H. Hematite; $F=$ Lattice of ferrites. Presence of gangue (dark grey) and pores (black).

Con este objeto se han propuesto varias vías de clasificación de los minerales. En una de ellas, los minerales se clasifican, según sus propiedades físicas, químicas y minerálogicas, en neutros $\left(\mathrm{Al}_{2} \mathrm{O}_{3}<\right.$ $2 \%)$, aluminosos $\left(\mathrm{Al}_{2} \mathrm{O}_{3}>2 \%\right)$, siliciosos $\left(\mathrm{SiO}_{2}>\right.$ $10 \%)$, concentrados y magnetíticos. Algunos operadores de las plantas de sinterización tienen su propia versión de esta clasificación. La característica es que habiendo decidido sobre una mezcla base, utilizando las distintas clasificaciones, sea posible intercambiar minerales sin que el producto final sufra modificaciones en cuanto a su calidad, manteniéndose los consumos de coque y la productividad. 
Estudio de la granulación de la mezcla de minerales de hierro...

Tabla VI. Análisis químico puntual de las fases identificadas en el sinterizado S30, \% en masa

Table VI. Point chemical analysis of the identified phases of sintering S2O, \% mass

\begin{tabular}{clrllllllll}
\hline $\mathbf{P}$ & Fase & \multicolumn{1}{c}{ Fe } & Ca & Al & \multicolumn{1}{c}{ Si } & Mn & Mg & Ti & K & O \\
\hline \multirow{2}{*}{53} & Ferrito & 44,25 & 8,29 & 0,72 & 2,19 & 0,78 & 0,28 & - & - & 43,49 \\
& Ganga & 9,21 & 22,68 & 0,25 & 12,55 & - & - & 0,42 & 0,22 & 54,67 \\
54 & Hematita & 59,07 & 0,29 & 0,20 & 0,21 & - & - & - & - & 40,23 \\
& Ferrito & 45,11 & 7,99 & 0,69 & 2,29 & 0,66 & 0,32 & - & - & 42,94 \\
& Ganga & 10,19 & 21,98 & 0,52 & 12,58 & - & 0,24 & - & 0,38 & 54,11 \\
57 & Hematita & 68,51 & - & 0,19 & - & - & - & - & - & 31,30 \\
& Ferrito & 56,14 & 5,87 & 0,61 & 0,94 & 1,20 & 1,19 & - & - & 34,05 \\
\hline
\end{tabular}

La clasificación de los minerales de hierro según su granulometría, ha seguido el siguiente proceso:

Antes de la aparición de los minerales concentrados el índice granulométrico utilizado era la fracción $<0,25 \mathrm{~mm}$, ya que en una primera época las granulometrías de todos los minerales eran muy semejantes y bastaba con caracterizar un punto de la curva para obtener toda la información del total de la misma.

Posteriormente, la media granulométrica D-50 y el tamaño medio fueron los índices utilizados. El primero se define como el índice que determina la mediana de la curva de distribución granulométrica de la mezcla; es decir, es el tamaño que deja, tanto por encima como por debajo, el mismo peso de mineral. Este índice intenta de alguna forma mejorar el pronóstico utilizando toda la curva granulométrica y no sólo un valor. Tiene el inconveniente de que valora con el mismo peso los tamaños finos que los gruesos, cuando los primeros tienen mayor importancia en sinterización.

El tamaño medio se obtiene ponderando el valor medio de cada uno de los intervalos en que se ha dividido la curva granulométrica por el peso del mineral contenido en cada intervalo granulométrico.

En un trabajo de colaboración entre ACERALIA y el CENIM, se establece un índice que se denomina "área de la curva granulométrica" ${ }^{\text {[15] }}$ que está relacionado con la finura del mineral. Se intenta dar a cada fracción de la curva granulométrica el peso que realmente tiene en la sinterización, para lo cual se utiliza el eje de abscisas con escala logarítimica, que da tanto más peso cuanto más fino es el tamaño de partícula. Se define este índice como el área encerrada entre la curva granulométrica del mineral y el eje de abscisas.

La Nippon Steel ha realizado un trabajo donde se define el índice $G$ para la clasificación de la granulometría de los minerales desde el punto de vista de su comportamiento en sinterización (ref. 9 de la I parte).
El índice $G$ viene definido por la expresión:

$$
\mathrm{G}=\frac{\mathrm{M} \times F}{N}
$$

donde $N$ representa el porentaje en masa de la fracción de partículas de tamaño $>0,7 \mathrm{~mm}$ de la curva granulométrica; $F$ la fracción $<0,2 \mathrm{~mm}$, y $\mathrm{M}$ la fracción comprendida entre las dos anteriores.

El índice $G$ ha sido establecido como resultado de la observación práctica de que algunas fracciones tienen un comportamiento positivo, frente a la granulación, mientras otras lo tienen negativo.

Así, la fracción $N$, de partículas de tamaño comprendido entre 0,7 y $8 \mathrm{~mm}$, constituye un excelente soporte para la formación de los gránulos; la fracción $F$, de tamaños inferiores a $0,2 \mathrm{~mm}$, por su finura se adhieren a las partículas de la fracción $N$ y son capaces de asimilarse formando gránulos estables. Sin embargo, la fracción $M$, constituida por partículas de tamaños entre 0,2 y $0,7 \mathrm{~mm}$, no es deseable puesto que las partículas no son suficientemente grandes para constituirse en núcleos estables, ni finas para ser adherentes. La fracción $M$, al quedar en libertad, se alojaría entre los huecos dejados por los gránulos perjudicando la permeabilidad del lecho y disminuyendo la productividad del proceso.

Las partículas de mineral se clasifican, según el índice $G$, en cuatro grupos: $A, B, C$ y $D$, en orden de finura descendente, donde el grupo A corresponde a los minerales gruesos y el grupo $\mathrm{D}$ al de los ultraconcentrados de gran finura.

Los límites establecidos para los grupos son los siguientes:

Grupo A: valor de $\mathrm{G} \leq 15$

Grupo B: valor de G 15-35

Grupo C: valor de G 35-70

Grupo D: valor de $G \geq 70$ 
La aplicación del índice $G$ a los minerales unitarios permite obtener una mezcla de minerales óptima para sinterizar, desde el punto de vista de la composición granulométrica. Otra aplicación, muy interesante, es la posibilidad de sustituir, unos por otros, minerales que estén dentro del mismo grupo de valores del índice $G$.

\subsection{Clasificación de los minerales por el índice $G$}

Los minerales de hierro forman parte de las mezclas minerales utilizadas para fabricar sinterizados, en las proporciones que se indican en la tabla VII. Con cada mezcla mineral se fabrica una serie de sinterizados. Las propiedades de los sinterizados se analizan en función de los minerales que entran en su composición y de los parámetros utilizados en la sinterización: altura del lecho, porcentaje de finos de retorno e índice de basicidad.

Tabla VII. Composición de las mezclas minerales, \% en masa

Table VII. Composition of the ore mixtures, \% mass

\begin{tabular}{lcccc}
\hline & $\mathbf{1}$ & $\mathbf{2}$ & $\mathbf{3}$ & $\mathbf{4}$ \\
\hline Andaluza & 4,0 & 10,3 & 10,3 & 10,3 \\
CVRD-Carajas & 25,0 & 27,8 & 21,3 & 24,4 \\
CVRD-Tubarao & - & - & 7,2 & 6,4 \\
San isidro & 20,0 & 7,2 & - & - \\
Mount Wright & 8,0 & - & 7,2 & 7,2 \\
SNIM-Tazadit & 8,5 & 10,3 & - & - \\
SNIM-Normal & 4,0 & 6,2 & - & - \\
Goa Silicioso & 6,5 & - & 10,3 & 10,3 \\
Nimco & 4,5 & - & - & - \\
Hamersley & 4,0 & 12,4 & - & 10,3 \\
MBR Brasil & - & 10,3 & 20,6 & 10,3 \\
Caliza & - & - & 7,9 & 6,1 \\
Lodos LD & 2,6 & 2,6 & 2,1 & 2,1 \\
Escoria LD & 3,0 & 3,0 & 2,1 & 2,1 \\
Chatarra & 1,3 & 1,3 & 2,1 & 2,1 \\
Polvo de tragante & 0,7 & 0,7 & 0,6 & 0,6 \\
Sínter finos de retorno & 7,9 & 7,9 & 8,1 & 8,1 \\
\hline
\end{tabular}

Los minerales de hierro se clasifican según el índice $\mathrm{G}$ en los siguientes grupos:

Grupo A: CVRD-Carajas, Hamersley, MBR Brasil Grupo B: Andaluza, CVRD-Tubarao, SNIMTazadit, SNIM-Normal, Nimco

Grupo C: San Isidro, Goa Silicioso

Grupo D: Mount Wright

Como se explica en el apartado siguiente, la experiencia práctica indica que los mejores resultados, para una buena granulación, se obtienen cuando para los valores del índice $G$ se cumplen las condiciones:

- Porcentaje de los minerales del grupo A y del grupo B en la mezcal mineral:

$$
A+B>68
$$

- Porcentaje de los minerales del grupo C y del grupo $\mathrm{D}$ en la mezcla mineral:

$$
\mathrm{C}+\mathrm{D}<12
$$

En la tabla VIII se indican los porcentajes de los minerales de hierro (Tabla VII) agrupados según el índice $G$ para cada mezcla mineral. De la observación de esta tabla se tiene que la mezcla 2 cumple las condiciones $\mathrm{A}+\mathrm{B}=77,3>68$ y $\mathrm{C}+\mathrm{D}=7,2<$ 12 , es decir, presenta un comportamiento ideal para la granulación. Las mezclas 3 y 4 no cumplen las condiciones anteriores y la mezcla 1 se aleja mucho de las condiciones ideales.

En la tabla VIII se ofrece el valor medio de la productividad para cada serie de sinterizados fabricados con cada mezcla mineral. Para comparación, se eligen las condiciones de operación utilizadas con las mezclas 3 y 4: altura de lecho, $60 \mathrm{~cm}$; finos de retorno, $25 \%$ e índice de basicidad 1,9 (ref. 26 de la I parte). Se puede observar que la mayor productividad se ha obtenido con la mezcla 2 (mejor

Tabla VIII. Minerales de hierro agrupados por el índice G, \% en masa

Table VIII. Iron ores classified as $G$ index, \% mass

\begin{tabular}{ccccccccc}
\hline $\begin{array}{c}\text { Mezcla } \\
\text { mineral }\end{array}$ & A & B & C & D & A+B & C+D & $\begin{array}{c}\text { Productividad } \\
\text { t/m2 } 2 \text { 24h }\end{array}$ & $\begin{array}{c}\text { Consumo de coque } \\
\mathrm{kg} / \mathrm{t} \text { de sínter }\end{array}$ \\
\hline 1 & 29,0 & 21,0 & 26,5 & 8,0 & 50,0 & 34,5 & 38,0 & 42,5 \\
2 & 50,5 & 26,8 & 7,2 & - & 77,3 & 7,2 & 40,4 & 42,4 \\
3 & 41,9 & 17,5 & 10,3 & 7,2 & 59,4 & 17,5 & 39,1 & 40,1 \\
4 & 45,0 & 16,7 & 10,3 & 7,2 & 61,7 & 17,5 & 39,4 & 40,5 \\
\hline
\end{tabular}


granulación), seguida de la 4 y 3 , y la productividad más baja la presenta la mezcla 1 .

Por otra parte el consumo más bajo de coque se obtiene con la mezcla 3 y el más alto con la mezcla 1 .

\subsection{Evolución de la composición de las parvas en ACERALIA}

En la tabla IX se recogen 30 minerales de hierro utilizados por ACERALIA en la banda de sinterización de $200 \mathrm{~m}^{2}$ de superficie, ordenados de más gruesos a más finos, clasificados según unos criterios diferentes: fracción de tamaño inferior a 0,125 $\mathrm{mm}$; área de la curva granulométrica; tamaño medio; D-50; índice G y grupo al que pertenecen por el valor del índice G. Se observa que los mine- rales se clasifican de forma semejante, independientemente del criterio escogido, y por ello se elige la clasificación según el índice G.

En las experiencias realizadas en paila, variando el índice G de 6 a 178, se ha observado que hay un óptimo en el consumo específico de coque que se corresponde con mezclas que contienen un porcentaje de minerales del grupo $\mathrm{A} \geq 50 \%$, del grupo $\mathrm{B}$ $\leq 35 \%$ (teniendo que cumplirse, $\mathrm{A}+\mathrm{B} \geq 68 \%$ ), del grupo $\mathrm{C} \leq 12 \%$ y del grupo $\mathrm{D} \leq 5 \%$ (necesariamente, $\mathrm{C}+\mathrm{D} \leq 12 \%$ ).

En la tabla X se ofrecen las mezclas utilizadas en la banda de sinterización en los últimos años, y la clasificación por grupos según el índice G. Se observa que las recuperaciones representan un porcentaje elevado en la mezcla $(15-23 \%)$ y están

Tabla IX. Clasificación de los minerales de hierro de acuerdo con el índice $G$

Table IX. Classification of iron ores as $G$ index

\begin{tabular}{|c|c|c|c|c|c|c|}
\hline & $\begin{array}{l}\text { \% fracción } \\
<0,125 \mathrm{~mm}\end{array}$ & Área & $\begin{array}{c}\text { Tamaño } \\
\text { medio, mm }\end{array}$ & D 50 & Índice G & Grupo \\
\hline Iscor & 6,0 & 215,9 & 2,55 & 2,28 & 2,30 & A \\
\hline BHP Yandi & 7,0 & 213,1 & 2,88 & 2,00 & 4,25 & $A$ \\
\hline Robe River & 8,1 & 213,2 & 3,06 & 2,14 & 6,13 & $A$ \\
\hline Hamersley & 14,1 & 195,7 & 2,30 & 1,67 & 9,63 & $A$ \\
\hline CVRD-Carajas & 11,7 & 197,0 & 2,32 & 1,57 & 10,11 & A \\
\hline MBR Brasil & 18,6 & 185,4 & 1,99 & 1,27 & 11,20 & A \\
\hline Samitri Brasil & 19,0 & 192,9 & 2,58 & 1,48 & 12,18 & $A$ \\
\hline Kiruna B & 20,0 & 169,7 & 1,23 & 0,76 & 12,51 & A \\
\hline Monut Newman & 16,0 & 185,6 & 1,95 & 0,94 & 12,96 & A \\
\hline Kiruna D & 20,0 & 187,0 & 2,31 & 1,43 & 13,28 & A \\
\hline Andaluza & 14,1 & 191,4 & 2,31 & 1,15 & 15,05 & B \\
\hline Romeral & 16,0 & 184,7 & 2,02 & 0,90 & 15,61 & B \\
\hline Samitri Sama & 19,0 & 186,2 & 2,41 & 1,18 & 17,78 & B \\
\hline SNIM-Tazadit & 27,0 & 180,8 & 2,61 & 1,11 & 18,88 & B \\
\hline CVRD-Silicioso & 26,0 & 171,6 & 1,88 & 0,79 & 19,85 & B \\
\hline CVRD-Tubarao & 25,7 & 171,7 & 1,82 & 0,78 & 20,05 & B \\
\hline Chow Hights & 25,0 & 177,4 & 2,35 & 0,82 & 20,19 & B \\
\hline SNIM-Acido & 30,2 & 166,8 & 1,94 & 0,63 & 22,63 & B \\
\hline Malberget & 13,0 & 156,5 & 0,64 & 0,47 & 29,38 & B \\
\hline Nmco & 11,5 & 182,8 & 2,15 & 0,57 & 30,11 & B \\
\hline Samitri Sili & 20,0 & 178,2 & 2,19 & 0,64 & 31,30 & B \\
\hline Chow Gule & 29,0 & 161,2 & 1,78 & 0,41 & 37,03 & C \\
\hline San Isidro & 17,6 & 172,5 & 1,92 & 0,46 & 37,80 & C \\
\hline Goa Silicioso & 27,1 & 156,3 & 1,56 & 0,33 & 53,64 & $C$ \\
\hline Guelbs Mauri & 28,0 & 135,3 & 0,51 & 0,30 & 68,34 & C \\
\hline Mount Wright & 13,0 & 143,5 & 0,44 & 0,33 & 94,93 & $\mathrm{D}$ \\
\hline Presur & 21,8 & 129,9 & 0,33 & 0,24 & 178,14 & $\mathrm{D}$ \\
\hline Carol lake & 31,0 & 116,5 & 0,27 & 0,19 & 282,09 & $\mathrm{D}$ \\
\hline Moirana & 33,0 & 108,0 & 0,19 & 0,17 & 3896,40 & $\mathrm{D}$ \\
\hline Morro Agudo & 65,0 & 80,8 & 0,11 & 0,22 & & $\mathrm{D}$ \\
\hline
\end{tabular}


Tabla X. Evolución de la composición de las parvas, \% en masa

Table X. Evolution of stockpiles composition, \% mass

\begin{tabular}{|c|c|c|c|c|c|c|c|c|c|c|c|c|c|}
\hline & Grupo & 1987 & 1988 & 1989 & 1990 & 1991 & 1992 & 1993 & 1994 & 1995 & 1996 & 1997 & 1998 \\
\hline Andaluza & B & 23,2 & 18,4 & 14,7 & 10,3 & 12,7 & 11,2 & 10,1 & 10,6 & 7,8 & 9,3 & 0,7 & - \\
\hline PRESUR & D & - & 0,8 & 1,8 & 2,1 & 2,3 & 1,6 & 1,7 & 1,7 & 1,2 & 1,7 & 0,2 & - \\
\hline Metal Química & B & 2,2 & 3,2 & 2,5 & 1,7 & 1,3 & - & - & - & - & - & - & - \\
\hline CVRD-Carajas & A & 6,6 & 9,4 & 12,0 & 19,1 & 22,7 & 27,4 & 26,4 & 26,0 & 30,8 & 26,5 & 35,8 & 34,1 \\
\hline Hamersley & A & 5,4 & 9,7 & 10,7 & 10,1 & 10,7 & 11,0 & 11,5 & 11,8 & 11,7 & 5,5 & 3,8 & - \\
\hline Indio Silicioso & C & - & - & - & - & 0,7 & 2,1 & 0,6 & 0,9 & - & - & 3,3 & - \\
\hline LINCO & C & - & - & - & 0,8 & 1,1 & 2,7 & 0,8 & & - & - & - & - \\
\hline MBR-Brasil & A & - & - & 1,0 & 4,0 & 6,7 & 5,1 & 10,9 & 9,2 & 3,5 & 8,7 & - & - \\
\hline Rober River & A & - & - & - & - & - & 2,0 & 3,1 & 4,1 & 7,7 & 9,6 & 10,9 & 11,2 \\
\hline NIMBA & A & 29,5 & 26,7 & 19,6 & 3,8 & - & - & - & - & - & - & - & - \\
\hline Río Doce & B & - & - & 3,2 & 6,8 & 0,9 & - & - & - & - & - & - & - \\
\hline Río Doce Silicioso & B & - & - & - & 2,0 & 5,0 & 5,4 & 9,5 & 1,4 & 0,5 & - & - & - \\
\hline San Isidro & C & 8,7 & 5,1 & 5,0 & 6,1 & 5,2 & 4,7 & 2,3 & 9,0 & 11,9 & 14,3 & 12,2 & 11,2 \\
\hline SNIM-Ácido & B & - & - & 0,7 & 3,5 & 3,4 & 2,3 & 3,8 & 4,4 & 5,5 & 4,2 & 6,0 & 6,8 \\
\hline SNIM-Tazadit & B & 2,8 & 6,3 & 7,5 & 6,2 & 3,7 & 3,5 & 1,1 & 4,7 & 3,8 & 4,6 & 5,2 & 3,3 \\
\hline Recuperaciones & - & 21,6 & 20,4 & 21,3 & 23,5 & 23,6 & 21,0 & 18,2 & 16,2 & 15,6 & 15,6 & 21,9 & 33,5 \\
\hline Grupo A & & 41,5 & 45,8 & 43,3 & 37,0 & 40,1 & 45,5 & 51,9 & 51,1 & 53,7 & 50,3 & 50,5 & 45,3 \\
\hline Grupo B & & 28,2 & 27,9 & 28,6 & 30,5 & 27,0 & 22,4 & 24,5 & 21,1 & 17,6 & 18,1 & 11,9 & 10,1 \\
\hline Grupo C & & 8,7 & 5,1 & 5,0 & 6,9 & 7,0 & 9,5 & 3,7 & 9,9 & 11,9 & 14,3 & 15,5 & 11,2 \\
\hline Grupo D & & - & 0,8 & 1,8 & 2,1 & 2,3 & 1,6 & 1,7 & 1,7 & 1,2 & 1,7 & 0,2 & - \\
\hline$\overline{A+B}$ & & 69,7 & 73,7 & 71,9 & 67,5 & 67,1 & 67,9 & 76,4 & 72,2 & 71,3 & 68,4 & 62,4 & 55,4 \\
\hline $\mathrm{C}+\mathrm{D}$ & & 8,7 & 5,9 & 6,8 & 9,0 & 9,3 & 11,1 & 5,4 & 11,6 & 13,1 & 16,0 & 15,7 & 11,2 \\
\hline \multicolumn{2}{|c|}{ Tamaño medio (mm) } & 2,26 & 2,30 & 2,20 & 2,19 & 2,18 & 2,23 & 2,15 & 2,13 & 2,19 & 2,18 & 2,20 & 2,22 \\
\hline \multicolumn{2}{|c|}{$>8 \mathrm{~mm}$} & 4,21 & 4,40 & 4,10 & 3,34 & 2,81 & 3,10 & 2,57 & 2,87 & 3,86 & 4,06 & 4,39 & 3,93 \\
\hline \multicolumn{2}{|c|}{$<0,125 \mathrm{~mm}$} & 11,88 & 10,90 & 11,90 & 11,71 & 11,18 & 10,80 & 11,27 & 11,74 & 11,53 & 10,33 & 10,93 & 9,45 \\
\hline
\end{tabular}

formadas por residuos de origen siderúrgico: cascarillas de laminación, lodos LD, polvo de tragante y finos de retorno, entre otros. En 1998 hay un porcentaje muy elevado de recuperaciones, un 33,5\%, y ello puede explicar el valor más bajo para $\mathrm{A}+\mathrm{B}$ $(55,4 \%)$ con relación a los años anteriores.

En la tabla XI se ofrece la evolución de la producción de sinterizados. Se observa un aumento en la productividad, de 30 a $38 \mathrm{t} / \mathrm{m}^{2} \cdot 24 \mathrm{~h}$, con excepción de una disminución en 1996, debida a paradas en la banda de sinterización ocasionadas por una menor demanda de sinterizados por los hornos altos.

Al relacionar la productividad con la granulometría, se observa en fábrica que existe una correspondencia entre la mejora en la composición granulométrica de la mezcla y el aumento de la productividad (Fig. 8) coincidiendo con el cambio en la composición de la mezcla utilizada y ajustando esta variación al criterio de valores del índice $G$, según las condiciones ideales, que vienen a refrendar en la práctica lo que de manera experimental se realizó en este trabajo.

El consumo de coque disminuye de 50 a $34 \mathrm{~kg} / \mathrm{t}$ de sínter. El descenso acusado en 1998 puede deberse al aumento en el porcentaje de las recuperaciones en la mezcla y que algún residuo integrado en las recuperaciones pueda haber aportado coque a la mezcla.

Los valores de los contenidos de hierro y de óxido ferroso se mantienen bastante estables en 56 $57 \%$ y en $5,4-6,1 \%$, respectivamente.

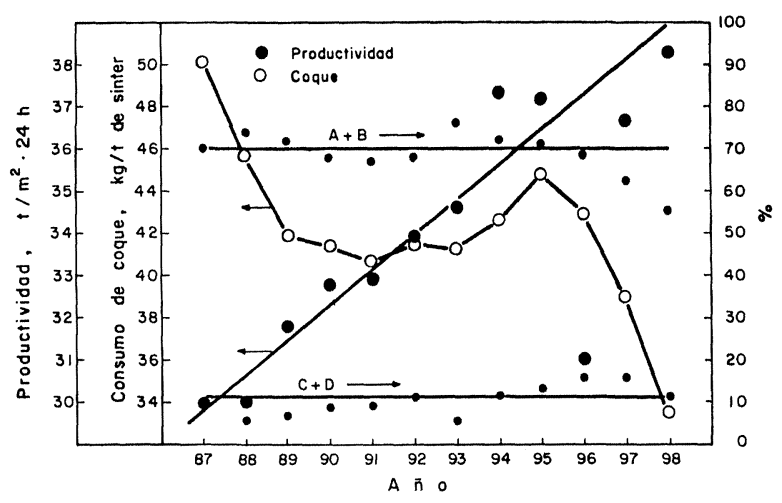

Figura 8. Productividad, consumo de coque y clasificación de los minerales de hierro, según el índice $G$, de la banda de sinterización.

Figure 8. Productivity, coke consumption and iron ores mixture classification, as $G$ index, of sintering strand. 
Tabla XI. Evolución de la producción de sinterizados

Table XI. Evolution of sinterings production

\begin{tabular}{|c|c|c|c|c|c|c|c|c|c|c|c|c|}
\hline & 1987 & 1988 & 1989 & 1990 & 1991 & 1992 & 1993 & 1994 & 1995 & 1996 & 1997 & 1998 \\
\hline Producción, t/24 h & 6.102 & 5.979 & 6.367 & 6.560 & 6.579 & 6.778 & 6.929 & 7.469 & 7.434 & 6.204 & 7.348 & 7.670 \\
\hline Product., $\mathrm{t} / \mathrm{m}^{2} \cdot 24 \mathrm{~h}$ & 30,1 & 29,9 & 31,8 & 32,8 & 32,9 & 33,9 & 34,6 & 37,4 & 37,2 & 31,0 & 36,7 & 38,3 \\
\hline Utilización,\% & 82,2 & 81,1 & 83,1 & 84,3 & 85,8 & 73,5 & 83,8 & 83,8 & 86,9 & 73,3 & 81,8 & 90,8 \\
\hline \multicolumn{13}{|l|}{ Consumo especiff, kg/t } \\
\hline Carga férrica & 979 & 995 & 988 & 985 & 989 & 990 & 988 & 979 & 977 & 985 & 962 & 983 \\
\hline Caliza & 111 & 111 & 111 & 108 & 106 & 113 & 117 & 127 & 127 & 133 & 122 & 109 \\
\hline Dunita & 24 & 5 & 24 & 24 & 22 & 25 & 16 & 28 & 31 & 33 & 36 & 34 \\
\hline Cal viva & 8 & 8 & 8 & 9 & 8 & 9 & 12 & 13 & 14 & 6 & 13 & 14 \\
\hline Dolomita calcinada & 7 & 8 & 8 & 7 & 8 & 8 & 6 & 4 & 4 & 3 & 3 & 4 \\
\hline Finos de retorno & 273 & 265 & 271 & 310 & 320 & 302 & 302 & 283 & 304 & 315 & 324 & 331 \\
\hline Coque & 50,1 & 45,7 & 41,9 & 41,6 & 40,7 & 41,8 & 41,3 & 42,6 & 44,7 & 42,9 & 38,9 & 33,5 \\
\hline \multicolumn{13}{|l|}{ Análisis químico, $\%$} \\
\hline Fe total & 55,81 & 56,71 & 56,33 & 56,77 & 56,78 & 56,98 & 56,95 & 56,91 & 56,99 & 57,24 & 56,95 & 56,53 \\
\hline $\mathrm{FeO}$ & 5,97 & 5,90 & 5,89 & 6,08 & 5,88 & 5,67 & 5,36 & 5,39 & 5,52 & 5,60 & 5,64 & 6,03 \\
\hline $\mathrm{SiO}_{2}$ & 6,12 & 5,62 & 5,88 & 5,90 & 5,73 & 5,58 & 5,46 & & 5,23 & 5,20 & 5,26 & 5,12 \\
\hline $\mathrm{Al}_{2} \mathrm{O}_{3}$ &, 39 & 1,36 & 1,42 & 1,41 & 1,43 & 1,50 & 1,39 & 1,49 & 1,50 & 1,39 & 1,31 & 1,38 \\
\hline $\mathrm{CaO}$ & 10,10 & 9,55 & 10,10 & 9,56 & 9,45 & 9,49 ， & 9,72 & 10,04 & 9,99 & 9,52 & 9,73 & 10,04 \\
\hline $\mathrm{MgO}$ & 1,67 & 1,68 & 1,61 & 1,62 & 1,62 & 1,57 & 1,64 & 1,53 & 1,61 & 1,69 & 1,92 & 1,80 \\
\hline $\mathrm{K}_{2} \mathrm{O}$ & 0,121 & 0,130 & 0,113 & 0,083 & 0,090 & 0,083 & 0,068 & 0,073 & 0,061 & 0,064 & 0,030 & 0,044 \\
\hline Basicidad, $\mathrm{CaO} / \mathrm{SiO}_{2}$ & 1,65 & 1,70 & 1,71 & 1,62 & 1,65 & 1,70 & 1,78 & 1,88 & 1,91 & 1,83 & 1,85 & 1,96 \\
\hline \multicolumn{13}{|l|}{ Ensayos, \% } \\
\hline$R D I(<3 \mathrm{~mm})$ & 28,2 & 28,9 & 29,3 & 33,9 & 29,5 & 31,5 & 35,6 & 37,8 & 38,4 & 40,3 & 33,4 & 31,7 \\
\hline Tumbler $(>6,35 \mathrm{~mm})$ & 76,3 & 75,1 & 75,0 & 74,6 & 74,2 & 74,7 & 75,4 & 75,3 & 75,7 & 76,8 & 75,3 & 75,0 \\
\hline Reductibilidad & 78,4 & 79,6 & 74,5 & 68,6 & 70,4 & 71,7 & 71,3 & 72,9 & 71,8 & 70,8 & 71,7 & 71,4 \\
\hline \multicolumn{13}{|l|}{ Granulometría } \\
\hline$>50 \mathrm{~mm}, \%$ & 7,05 & 6,70 & 7,40 & 8,60 & 8,30 & 10,00 & 7,10 & 7,00 & 5,97 & 7,23 & 5,87 & 3,06 \\
\hline $10-5 \mathrm{~mm}, \%$ & 18,14 & 17,30 & 19,60 & 16,70 & 15,30 & 12,10 & 7,30 & 7,40 & 6,83 & 7,40 & 10,55 & 12,90 \\
\hline$<5 \mathrm{~mm}, \%$ & 7,81 & 8,00 & 7,70 & 7,70 & 8,30 & 7,60 & 8,50 & 7,20 & 4,63 & 5,02 & 5,72 & 6,25 \\
\hline Tamaño medio, mm & 21,83 & 21,70 & 21,57 & 23,27 & 23,27 & $2 \quad 4,73$ & 24,55 & 24,88 & 24,56 & 24,26 & 23,37 & 20,28 \\
\hline
\end{tabular}

Para los índices de calidad de los sinterizados, el valor del RDI aumenta (empeora) de 28 a $40 \%$ y en los dos últimos años disminuye (mejora) al 32 $\%$. El índice Tumbler oscila entre 74 y $77 \%$. La reductibilidad oscila entre 69 y $80 \%$.

El tamaño medio de los sinterizados se mantiene entre 20 y $25 \mathrm{~mm}$, tamaño adecuado para ser cargado al horno alto.

\section{CONCLUSIONES}

El estudio de la granulación de mezclas minerales, utilizadas para fabricar sinterizados, realizado con datos obtenidos en laboratorio, planta piloto y planta industrial, permite establecer las siguientes conclusiones.

- Los minerales de hierro y las mezclas minerales presentan una buena aptitud a la granulación.

- Se ha obtenido la estructura óptima de cuasipartícula en el sinterizado, formada por un núcleo de hematita rodeado por una red de ferritos aciculares. Esta estructura se ha conseguido como resultado de una buena granulación.

- Se ha definido el índice de granulación (índice G) y se ha utilizado para clasificar una serie de treinta minerales de hierro.

- Con la mejora del índice G, que se consigue con una elección adecuada de los componentes de la mezcla mineral, se observa un aumento en la productividad y un descenso en el consumo de coque en la banda de sinterización de ACERALIA. La ley en hierro y los índices de calidad de los sinterizados no experimentan un cambio apreciable.

\section{Agradecimientos}

Los autores agradecen a ACERALIA Corporación Siderúrgica la información facilitada y a $\mathrm{D}$. Clemente Cubillo el apoyo experimental para la realización de este trabajo. 


\section{REFERENCIAS}

[1] A. FORMOSO y J. MOCHÓN, Improvement of the yield of sinter strand and the regularity of sinter by control of charging conditions and monitoring of the flame front propagation. ECSC Agreement No. 7210-PR003. 1997.

[2] M.T. LARREA, A. Formoso, M. SiRgado, A. GUTírRez, P.P. GÓMEZ y A. CORES, Ironmaking Steelmaking, 19 (1992) 311-319.

[3] A. CoRES, A. Formoso, M. SIRGADO, J.L. VERDURAS Y L. CALEA, Ironmaking Steelmaking, 23 (6) 1996: 486-492.

[4] A. Cores, A. Formoso, A. Moro y P. Marañón, Rev. Metal. Madrid 32 (1996) 25-32.

[5] A method for determining the low temperature desintegration behaviour of iron ores, pellets and sinters by cold tumbling after static reduction. Third draft, ISO Proposal, ISO/TC 102/SC 3/285 E, 1974.

[6] H.A. KORTMAN y BURGHARDT, Agglomeration 77. Ed. Sastry, K.V.S., AlME, New York, 1977, pp. 219-231.
[7] P.W. ROLleR, Trans. Iron Steel Inst. Jpn. 26 (1986) 834835.

[8] Standard method for tumbler test for iron ores, pellets and sinters. Designation: E 279-69 (Reaproved 1979).

[9] C.E. LOO, K.T. WAN y V.R. HOWES, Ironmaking Steelmaking, 15 (1998) 279-285.

[10] E. KASAI, S. WU e Y. OMORI, ISIJ Int. 31 (1991) 17-23.

[11] E. KaSAI, W.J. RANKIN, R.R. LOVEl e Y. OMORI, ISIJ Int. 9 (1989) 635-641.

[12] T. MAEDA Y T. ONO, Trans. Iron Steel Inst. Jpn. 25 (1985) 1191-1193.

[13] S.N. AHSAN, T. MUKHERJEE y J.A. WhITEMAN, Ironmaking Steelmaking 10 (1983) 54-64.

[14] T. MUKHERJEE y J.A. WHITEMAN, Ironmaking Steelmaking 12 (1985) 151-155.

[15] M. SiRgado y E. Ferrón, Resúmenes de Comunicaciones, IV Asamblea General del CENIM, núm. 37. Madrid, 31 mayo-2 junio, 1970. 